International Journal of Engineering \& Technology, $7(2.19)(2018)$ 106-110
International Journal of Engineering \& Technology
SPC
Website: www.sciencepubco.com/index.php/IJET
Research paper

\title{
Satellite Image Registration and Image Fusion by using Principle Component Analysis
}

\author{
Gandla Maharnisha ${ }^{1}$,Gandla Roopesh Kumar $^{2}$,R.Arunraj ${ }^{3}$ \\ ${ }^{1} U G$ Scholar \\ Department of Computer Science and Engineering, School of Computing, \\ Vel Tech Rangarajan Dr. Sagunthala R\&D Institute of Science and Technology, \\ Avadi, Chennai-600 062, Tamil Nadu, India. \\ ${ }^{2} U G$ Scholar, Department of Electronics and Communication Engineering \\ Jawaharlal Nehru Technological University \\ Chittoor, India \\ ${ }^{3}$ Assistant Professor \\ Department Of Automobile Engineering, \\ Vel Tech Rangarajan Dr. Sagunthala R\&D Institute of Science and Technology, \\ Avadi, Chennai-600 062, Tamil Nadu, India. \\ *maharnisha29@gmail.com
}

\begin{abstract}
This aims to fused image registration and image fusion used to spatial resolution images by principle component analysis method. Digital image processing requires either the full image or a part of image. It will be processed from the user's point of view like the radius of object. Wavelet technique will improve the spatial resolution to produce spectral degradation in output image. To overcome the spectral degradation, PCA fusion method can be used. PCA uses curve which represent edges and extraction of the detailed information from the image.PAN and MS images are used by individual acquired low frequency approximate component and high frequency detail components in this PCA. To evaluate the image fusion accuracy, Peak Signal to Noise Ratio and Root Mean Square Error are used. The advantages of using digital image processing are preservation of original data accuracy, flexibility and repeatability.
\end{abstract}

Keywords: Image Enhancement; Image Registration; Image Fusion; PCA;Remote sensing

\section{Introduction}

The remote sensing is gaining huge popularity and as a continuously growing market with applications like vegetation mapping and observation of the environment. The reasons for the wide range of application are by the availability of high quality images for a reasonable price and computation power. There is a need for higher classification accuracy and enhanced positioning precision as the demand has increased. It has needed to improve the spectral and spatial resolution of remotely sensed imagery. Building new satellites with a superior resolution power or by the utilization of image processing techniques. The basic advantages are: the second alternative is the expenses are lower. Image fusion is a process which is used for integrating relevant information from two or more images into a single image.

\section{Need for the Study}

Image fusion implementsin different types of wavelets with their hard and soft threshold values to get better results. For efficiency results it is implemented on real time videos and images with high resolution capture devices. Weare planningto fuse more than two images to produce a de-blurred fused image for future results. Here digital system is majorly applied in different areas, for producing good contrastin digital images and strong in demand especially inthese areas such as computer vision, remote sensing, identification of models at huge level and fault detection.

\section{Description}

3.1 Image fusion Levels

The image fusion levels are:

1. Pixel

2. Feature

3. Block or Region

* Pixel: This is done by lower level in image fusion at the one of the simplest technique. The integrating values and intensities of two input images founded on the average and give the single resultant image by pixel levels.

* Feature: It justifies the features of image like distorted eye, 
distorted head and nose. In this techniqueit easily extracts the features of both similar images individually, and then fusion algorithm gives the enhanced image after extraction of the feature.

- Block or region : In block or region take place to the pixel blocks of the imagefusion. It is one of the highest level techniques and represented by multistage and measured values are calculated based on the regions.

\subsection{Image Fusion Types}

In this research to successfully bring about optimum fusion results and various wavelet-based fusion schemes to be tested.

\subsubsection{Intensity-hue-saturation for image fusion}

* The major steps involved in the remote sensing applications are: Fusion of panchromatic image and multispectral satellite images. In intensity the saturation fusion method, a colour image from the red, green and blue is converted into the HIS Colour space. The intensity band in the IHS colour space is replaced by a high-resolution pan image and then it is converted again to the original colour space by combining with the previous hue $(\mathrm{H})$ band and saturation band which finally results in an IHS fused image.

* Steps for IHS transform fusion image

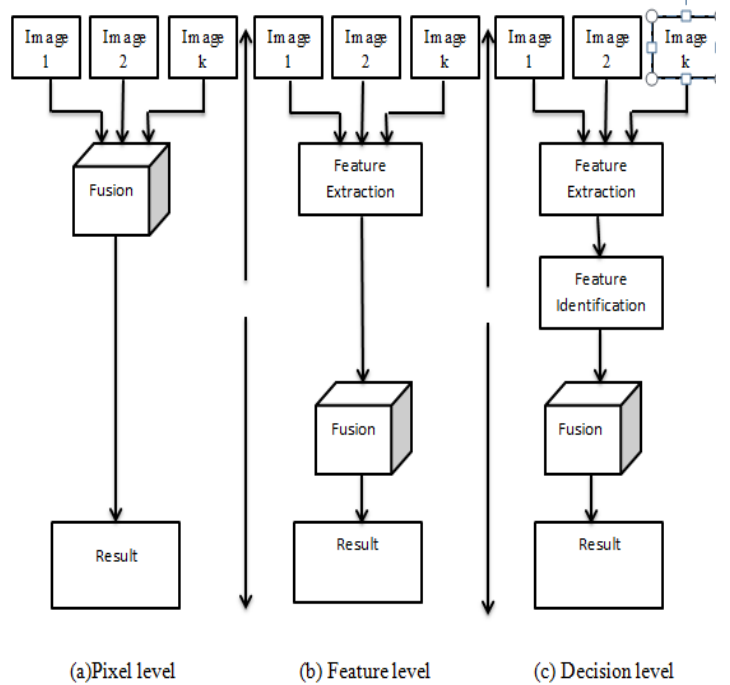

Fig.1: Image fusion levels technique

Step1: The IHS fusion for each pixel can be formulated.

Step2: The intensity component I is replaced by the Pan image. Step3: The fused image $[\mathrm{F}(\mathrm{R}) ; \mathrm{F}(\mathrm{G}) ; \mathrm{F}(\mathrm{B}] \mathrm{T}$ can be easily obtained from the original image [R; G; B] T simply by using addition operations.

The improved IHS transform converts an RGB image into spatial (I) and spectral $(\mathrm{H}, \mathrm{S})$ information by reducing the color distortion. The IHS fusion method adopts color distortion when it is processing IKONOS images.

\subsubsection{Brovey Transform}

Pixel level image fusion is introduced byBroveytransform.Brovey used to perform a transformation by three multispectral and panchromatic satellite image scene channels. It is also called as color normalization transform, because it involves in a red-green -blue (RGB) color transform method.

\subsubsection{High-Pass Filtering Method}

They are usedin digital image processing forimage modifications, enhancements, noise reduction byusing designs that are done in either spatial domain or frequency domain.

\subsection{Remote Sensing Image Fusion}

The several applications involved in the remote sensing domains which are: multi-resolution image fusion, it is one of the important domains in remote sensing. Panchromatic images are collected in the broad visual length. But that one rendered in black and white. Another one type of multispectral images obtains in extra one spectral or wave length.

\subsubsection{Brovey Transform}

Pixel level image fusion is introduced byBroveytransform.Brovey used to perform a transformation by three multispectral and panchromatic satellite image scene channels. It is also called as color normalization transform, because it involves in a red-green -blue (RGB) color transform method.

\subsubsection{High-Pass Filtering Method}

They are usedin digital image processing forimage modifications, enhancements, noise reduction byusing designs that are done in either spatial domain or frequency domain.

\subsection{Remote Sensing Image Fusion}

The several applications involved in the remote sensing domains which are: multi-resolution image fusion, it is one of the important domains in remote sensing. Panchromatic images are collected in the broad visual length. But that one rendered in black and white. Another one type of multispectral images obtains in extra one spectral or wave length

\subsection{Satellite Image Fusion}

"Data fusion" has been adopted and widely used since 1990's. For example, it deals with data and information from multiple sources to achieve refined/improved information for decision making.

In this fusion we have two input images; these both images are same but does not have relevant information about image. So, itfuses both the images to make it relevant in the output image for both multispectral as well as panchromatic images. Both input images will get good resolution in output image.

It mostly uses in the medical areas very effectively. It is a general approach to extract information from various images and it willtransform it to an informatics image. The aim of image fusion (IF) is to get quality of image by combining complementary multisensoryand multi temporal into one new image which contains information.

\subsection{Image Registration}

To transform various sets of data into one co-ordinate system is referred as 'Image registration'. Data may be multiple photographs, different sensors, in different times in different viewpoints. It is widely used in computer vision, medicalimaging, military automatic target recognition, compiling and analyzing images and data from the satellites. To determine atransformation of most accurate match between two images. This matching transformation is very time- consuming and tedious. With the increase of data computational time is evencritical. It is used in remote sensing applications; therefore,automatic image registration is needed.

\section{Literature Review}

Cle Pohl described about more available of multisensor,multi temporal,multiresolution image data based operational earth observation satellites the fusion of digital image data. It one describes and explains based image fusion process of earth observation satellite data as a contribution to multisensory integration oriented data processing. [1] 
Maria Gonzalez reviewed about high pass filtering procedure to fuse multispectral and panchromatic images and different fusion level has been improved based on same principle to follow extract from the panchromatic image spatial information. After that spatial information to later inject it into the multispectral one. It is using by the principle component analysis method to compute wavelet decomposition.[2]

Shuyuan Yang described improving spatial resolution by structure concept assumption for fusion of panchromatic and multispectral images. First it one support value filter(SVF) is used to founded by new multiscale model(MSM),support vector transform(SVT), and adaptive principle component analysis(APCA) is then employed to select the principle component analysis method.[3]

\section{Architecture and Design}

\section{List of modules}

* Image De-noising

* Image Enhancement

* Image Registration

* Image Fusion

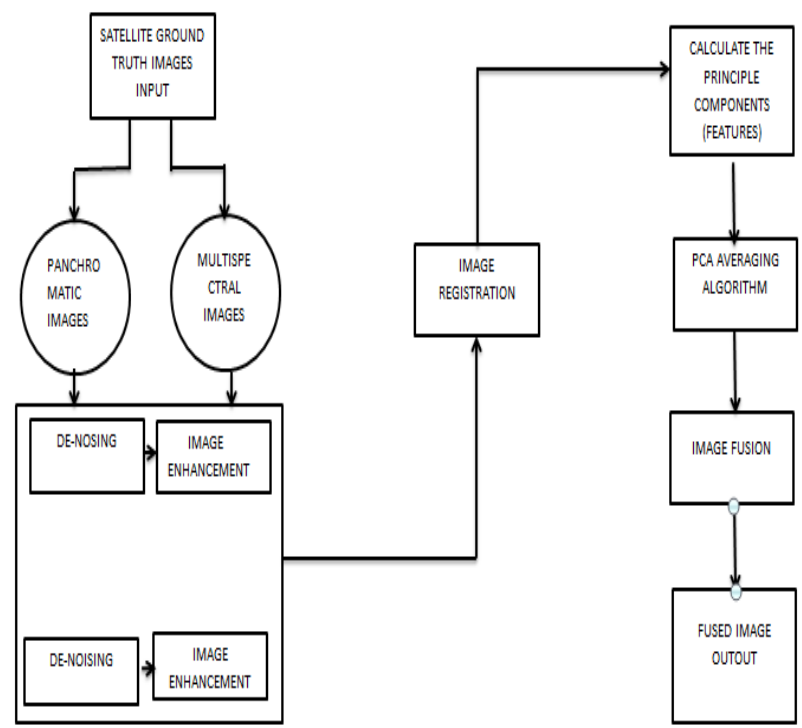

Fig.2: Architecture of Image Fusion

\subsection{Image De-Noising Using Adaptive Median Filter- ing}

Image De-noising using Adaptive Median filter algorithm based on the median value in a window corrupted pixels are identified and restored.

\section{Algorithm}

Step1: Image Acquisition

Step2: Apply Adaptive Median Filter for both Grey and Colour space Images

Step3: Subtract the Filtered Image from Input Image

Step4: Calculate the Noisy Pixels

\subsection{Image Enhancement Using Mean Adjustment}

Input image enhancement using mean adjustment (Mean adjustment is used to improve the contrast, brightness).
Step2: Case 1: PAN Scale Image

Step3: Set the thresholding values (Median)

Step4: Set lower and upper thresholding values to calculate the Minima and Maxima.

Step5: Apply the double Precision to the Image

Step6: Apply Normalization

Step7: Calculate the Mean of the Gray Scale Value

Step8: Adjust the Mean Value

Step9: Case 2: Multispectral Image

Step10: Set lower and upper thresholding values to calculate the Minima and Maxima

Step11: Color Image thresholding (Image Bandwidth)

Step12: Convert from Multispectral RGB Image to NTSC Color format

Step13: Calculate the Mean adjust value for Green layer using Color Image Upper thresholding

Step14: Calculate the mean adjust value for blue layer using color image lower thresholding

Step15: For Case 1 and Case 2:mean adjustment for first layer calculate minima and maxima

Step16: Apply formula (Image-Minima/Maxima-Minima)

Step17: Enhanced Image Output.

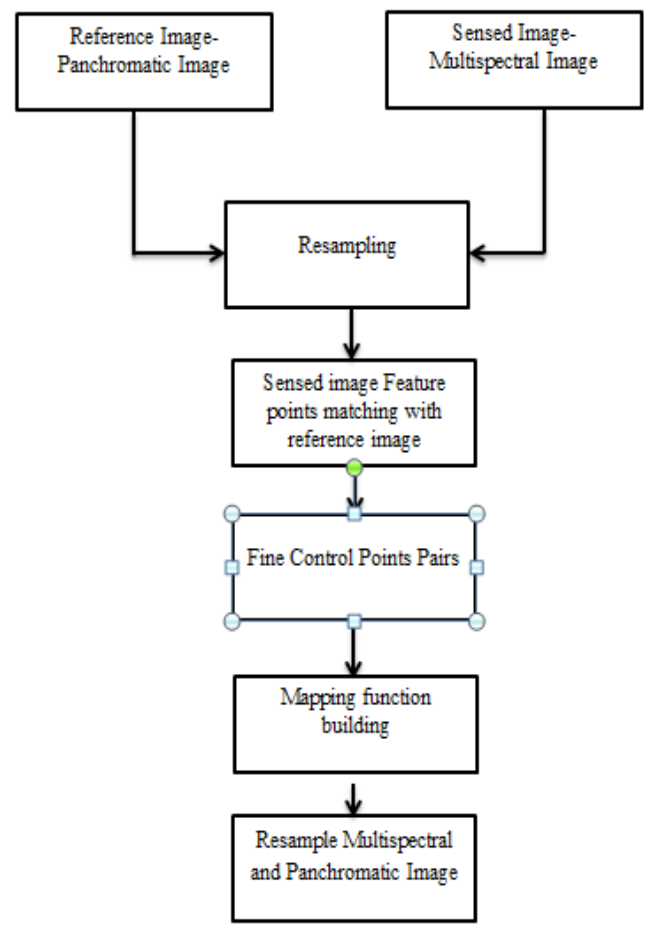

Fig.3:Flow diagram of image registrationalgorithm

\subsection{Image Registration Using Resampling}

Arranging two or more images of the same scene in that process is called image registration. It involves designating one image as the reference and applying geometric transformations to the other images as reference to align process by image registration.Re resizing the low resolution multispectral image to

\section{Algorithm}

Step1: Input the Filtered Image 
the same size in that process combining the panchromatic image

\section{Algorithm}

Step1: Feature extraction-Identifies the applicable features in the two images (Reference image, sensed image).

Step2: Feature matching-In this relationship between the features in the Multispectral and panchromatic mages.

Step3:Mapping function building - It determines the transformation parameters of the mapping functions using the features being matched and during the process of finding control points pairs, three bands multispectral image first is turned into one band gray image.

Step4: Image registration - Resampled Multispectral Image.

\subsection{Image Fusion}

Image Fusion techniques for combining a high-resolution panchromatic image also with a multispectral image. The fused image carrying both high-resolution spatial information of the panchromatic image and color information of the Multispectral image to get more results. A high resolution of multispectral images produces from the techniques details of panchromatic image and color information of Multispectral images.

\section{Principal component analysis for image fusion}

Principal component analysis is based upon the variable data sets and analysis for image fusion. In this datasets the variables are agree and disagree to around axis in linear transformation from measure space to feature space. It is basically pixel level fusion type levels of image fusion and multidimensional sets to lower level dimensions by measuring weight. The image fusion using Eigen vector preferably than picking the largest Eigen value.

\section{$\mathrm{Y}=A T \mathrm{X}$}

Where A is the matrix of normalized Eigen vector of covariance matrix of $\mathrm{X}$, and $\mathrm{Y}$ has a diagonal covariance matrix

\section{PCA algorithm}

Actually in this work we are fusing two images, both having some part blurred. So to get high quality image from these two input images we are adapting an popular Image Fusion Technique named PCA (Principal Component Analysis).

\section{Steps are as follows}

1. Read two images

2. Get mean of both images

3. Subtract mean from each element of an image (in both images).

4. Get the covariance for both images

5. Get Eigen values and Eigen Vector

6. Rearrange the Eigen vector using Single Value Decomposition

7. The values of 1 st column are principal coefficients

8. Multiply first coefficient with 1 st image and second coefficient with second image and add both images.

\section{Requirement Specification}

The purpose of using high-specification MATLAB software are visualization ,technical computing, integrates computation, and programming is faster to find out the problems and solutions than other software which are expressed in familiar mathematical elements, data exploration ,acquisition, analyzing and visualization, Engineering drawing and scientific graphics, mathematical functions and computational functions, Similarity problems prototyping and modeling, application development programming using GUI building environment.
Using Mat Lab, solution of computing problems and solving quickest than the traditional programming languages. The programming languages are $\mathrm{C}, \mathrm{C}++$.

Table 1: Mat lab requirements

\begin{tabular}{|l|l|l|} 
Table 1: Mat lab requirements \\
\begin{tabular}{|l|l|l|}
\hline $\begin{array}{l}\text { Software Require- } \\
\text { ments }\end{array}$ & $\begin{array}{l}\text { Hardware Re- } \\
\text { quirements }\end{array}$ & Platform \\
\hline MATLAB 2014 & $\begin{array}{l}\text { Processor - i3 } \\
\text { Version 7.12 }\end{array}$ & Windows/Linux \\
& $\begin{array}{l}\text { Hard Drive }-500 \\
\text { GB }\end{array}$ & \\
& & \\
\hline
\end{tabular}
\end{tabular}

\section{Result and Discussions}

Table 2: Comparison Chart

\begin{tabular}{|l|l|l|l|}
\hline Parameters & Images & $\begin{array}{l}\text { Proposed Fu- } \\
\text { sion method }\end{array}$ & Values \\
\hline \multirow{2}{*}{ PSNR } & $\begin{array}{l}\text { Panchromatic } \\
\text { Image and } \\
\text { Multispectral } \\
\text { Image }\end{array}$ & $\begin{array}{l}\text { Average } \\
\text { PCA(Principal } \\
\text { Component } \\
\text { Analysis) }\end{array}$ & 65.0455 \\
\cline { 1 - 1 } RMSE & $\begin{array}{l}\text { Panchromatic } \\
\text { Image and } \\
\text { Multispectral } \\
\text { Image }\end{array}$ & & $1.5380 \mathrm{e}-05$ \\
& & & \\
\hline
\end{tabular}

PSNR

Peak Signal to Noise Ratio (PSNR) is used for measurement of quality between the two images such as original and a reconstructed image. By measuring the quality of reconstructed image has higher using PSNR. To compute the PSNR, first we have to compute the mean squared error (MSE).So, there are some following equations:

Mean Square Error

$$
M S E=\frac{1}{m n} \sum_{i=0}^{m-1} \sum_{j=0}^{n-1}[I(i, j)-K(i, j)]^{2}
$$

Peak Signal-to-Noise Ratio

$$
P S N R=10 \cdot \log _{10}\left(\frac{M A X_{I}^{2}}{M S E}\right)
$$

\section{Image enhancement}

PSNR for Hyperspectral image...

49.6668

MSE for Hyperspectral image...

2.1367e-04

PSNR for PAN image...

43.3438

MSE for PAN image...

4.4247e-04

Image enhancement

PSNR for Fusion image...

65.0455

MSE for Fusion image...

\section{$1.5380 \mathrm{e}-05$}

\begin{tabular}{|c|c|c|c|c|c|c|}
\hline \multirow{3}{*}{ IMAGE NAME } & \multicolumn{6}{|c|}{ PCA Fusion } \\
\hline & \multicolumn{2}{|c|}{$\begin{array}{l}\text { Without En- } \\
\text { hancement }\end{array}$} & \multicolumn{2}{|c|}{$\begin{array}{l}\text { With AMF En- } \\
\text { hancement }\end{array}$} & \multicolumn{2}{|c|}{$\begin{array}{l}\text { With Hybrid } \\
\text { Enhancer }\end{array}$} \\
\hline & PSNR & RMSE & PSNR & RMSE & PSNR & RMSE \\
\hline $\begin{array}{l}\text { Image 1-Fused } \\
\text { Image }\end{array}$ & 63.43 & 0.0102 & 64.04 & 0.0101 & 66.45 & 0.0095 \\
\hline Image 2-Fused & 56.25 & 0.0161 & 61.22 & 0.0126 & 63.77 & 0.0102 \\
\hline
\end{tabular}

Table 3:PSNR and RMSE Comparison for PCA Image Fusion 


\begin{tabular}{|l|l|l|l|l|l|l|}
\hline Image & & & & & & \\
\hline $\begin{array}{l}\text { Image 3-Fused } \\
\text { Image }\end{array}$ & 64.24 & 0.0118 & 65.15 & 0.0095 & 66.45 & 0.0088 \\
\hline $\begin{array}{l}\text { Average of } \\
\text { Fusion Results }\end{array}$ & $\mathbf{6 1 . 3 0}$ & $\mathbf{0 . 0 1 2 7}$ & $\mathbf{6 3 . 4 7}$ & $\mathbf{0 . 0 1 0 7}$ & $\mathbf{6 5 . 5 5}$ & $\mathbf{0 . 0 0 9 5}$ \\
\hline
\end{tabular}

\subsection{Performance analysis}

It shows the experimental results of PCA various image enhancements and without image enhancement of PSNR and RMSE respectively. Fig.4 and Fig.6 show the plots derived from PSNR and RMSE various values of Principle component analysis image fusion. Fig.5 and Fig.7 show average values of PSNR and RMSE values of PCA image fusion.

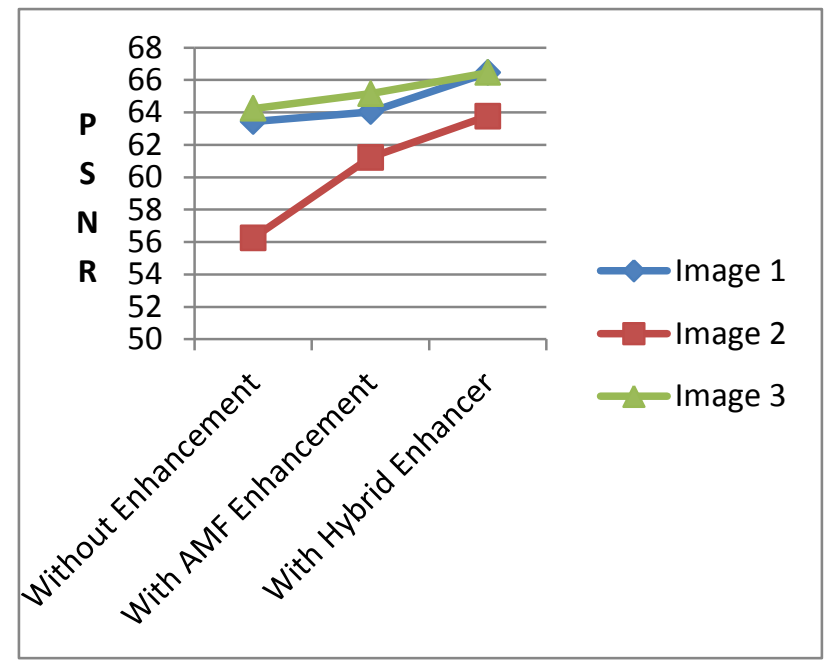

Fig.4: PSNR value for PCA various image fusion

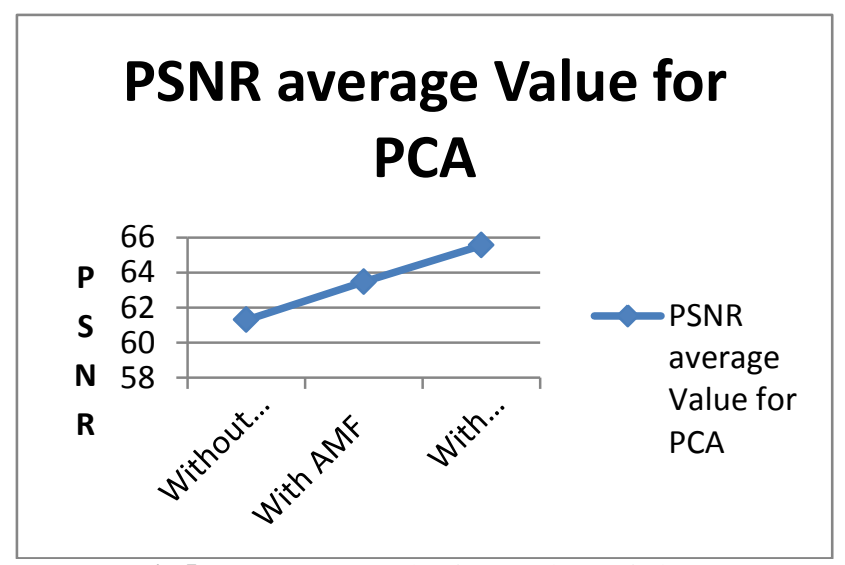

Fig.5:Average PSNR value for PCA image fusion

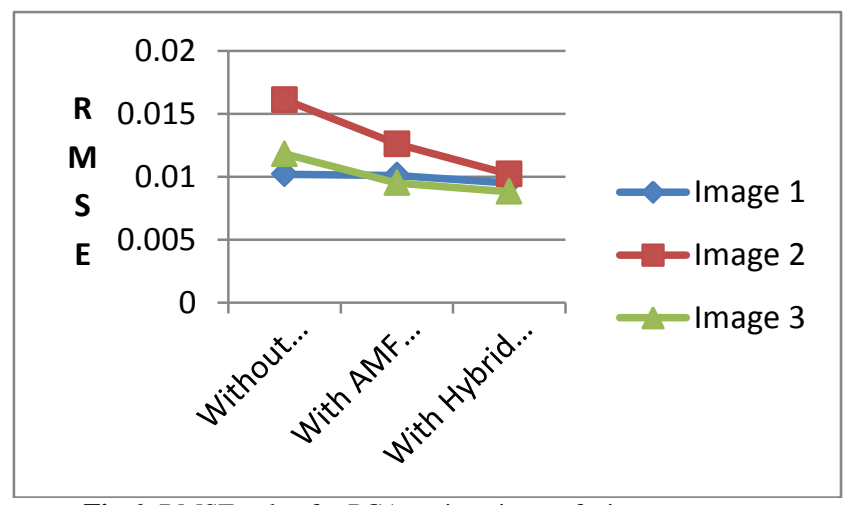

Fig.6: RMSE value for PCA various image fusion

\section{RMSE average value for PCA}

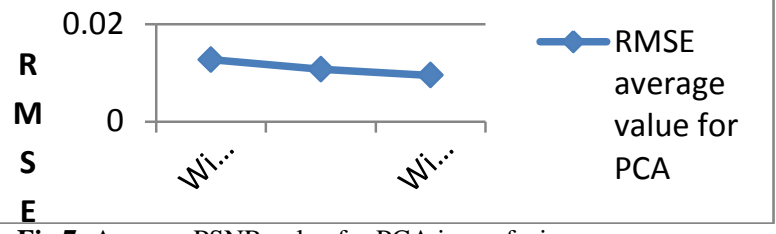

Fig.7: Average PSNR value for PCA imagefusion

\section{Conclusion}

Image fusion is the process which combines the information from all groups of images of same size. This results in generating new images that have the contents and the most desirable information of the input image. This finds the main application in two areas which are merging the gray-level high-resolution panchromatic image and the colored low resolution multi spectral image. The fusion appears in the color images and gets the color distortion. It is human visualization and evaluation criteria related problems when the image fusion occurred between the two images. Finally, the color distortion image fusion is implemented and getting the outcome problems. In this paper, given the results of the image fusion and image registration is founded from Principle component Analysis (PCA). A new image that employs the high-spatial resolution of images and the color information of the Multispectral images is generating by image fusion and image registration. In this paper evaluated the different parameters like Peak Signal to Noise Ratio (PSNR) and Root Mean Square Error (RMSE) was founded by principle component analysis method based fusion images and fusion registration.

\section{References}

[1] Cle Pohl,JL Van Genderen, "Multisensor image fusion in remote sensing:concepts,methods and applications,"International journal of remote sensing, Vol. 19,no.5,pp.823-854,1998.

[2] Maria Gonzalez-Audicana,Jose Luis Saleta,"Fusion of multispectral and panchromatic images using improved IHS and PCA mergers based on wavelet decomposition,"IEEE transactions on Geoscience and Remote sensing, Vol.42,no.6,pp.1291-1299,2004.

[3] Shuyuan Yang,Min Wang,Lincheng Jiao,"Fusion of multispectral and panchromatic images based on support value transform and adaptive PCA,"Information Fusion,Vol.13,no.3,pp.177-184,2012.

[4] Deepak kumar Sahu,MP Parshal,"Different image fusion techniques,"International journal of modern Engineering Research, $\mathrm{Vo}$ 2,no.5,pp 4289-4301,2012.

[5] Te-Ming Tu,Ping Sheng Huang,Chung-Line Hung,Chien-Ping Chang,"A fast intensity-hue-saturation fusion techniquewith spectral adjustment for IKONOS imagery,"IEEE Geoscience and Remote sensing,Vol 1,no.4,pp 309-312,2004.

[6] Jorgenunez,Xavier Otazu,Ocatavi Fors,"Multiresolution-based image Fusion with additive wavelet decomposition,"IEEE Transactions on Geoscience and Remote sensing,"Vol.37,no.3,pp.1204-1211,1999.

[7] Bruno Aiazzi,Luciano Alparone,"Context-driven fusion of high spatial and spectral resolution images IEEE Transactions on Geoscience and based on oversampled multiresolution analysis," Remote sensing,"Vol.40,no.10,pp.2300-2312,2002.

[8] Myungjin Choi,"A new intensity-hue-saturation fusion approach to image fusion with a tradeoff parameter,"IEEE Transactions on Geoscience and Remote sensing,"Vol.44,no.6,pp.1672-1682,2006.

[9] P.Geetha, B.Chitradevi,"De-noising Using Adaptive Weighted Median Filter with Synthetic Aperture Radar Images,"vol. 2, issue. 3, pp.413420,201 